\title{
Volatility Spillover Dynamics between Large-, Mid-, and Small-Cap Stocks in the Time-Frequency Domain: Implications for Portfolio Management
}

\author{
Sangram Keshari Jena ${ }^{1, *}$, Aviral Kumar Tiwari ${ }^{2}{ }^{\mathbb{D}}$, Ashutosh Dash ${ }^{3}$ and Emmanuel Joel Aikins Abakah ${ }^{4}$ \\ 1 Finance \& Economics, International Management Institute, Bhubaneswar 751003, India \\ 2 Finance \& Economics, Rajagiri Business School, Rajagiri Valley Campus, Kochi 682039, India; \\ aviral.eco@gmail.com \\ 3 Accounting \& Finance, Management Development Institute, Gurugram 122007, India; ashutosh@mdi.ac.in \\ 4 Department of Applied Economics, University of Cape Coast, Cape Coast CC-075-8216, Ghana; \\ ejabakah@gmail.com \\ * Correspondence: drsangramkjena@gmail.com
}

Citation: Jena, Sangram Keshari, Aviral Kumar Tiwari, Ashutosh Dash, and Emmanuel Joel Aikins Abakah. 2021. Volatility Spillover Dynamics between Large-, Mid-, and Small-Cap Stocks in the Time-Frequency Domain: Implications for Portfolio Management. Journal of Risk and Financial Management 14: 531. https://doi.org/10.3390/jrfm14110531

Academic Editors: Thanasis Stengos and Shigeyuki Hamori

Received: 3 September 2021

Accepted: 18 October 2021

Published: 8 November 2021

Publisher's Note: MDPI stays neutra with regard to jurisdictional claims in published maps and institutional affiliations.

Copyright: (c) 2021 by the authors. Licensee MDPI, Basel, Switzerland. This article is an open access article distributed under the terms and conditions of the Creative Commons Attribution (CC BY) license (https:// creativecommons.org/licenses/by/ $4.0 /)$.

\begin{abstract}
The connectedness dynamics between large-, mid-, and small-cap stocks is investigated using the forecasted error variance decomposition (FEVD) spillover framework of Diebold and Yilmaz in the time-frequency domain. Total volatility spillover (i.e., connectedness) is elevated between large-, mid-, and small-cap stocks during the study period. This high level of spillover exists in the short run only, and declines gradually in the medium to long run, thus providing opportunities for portfolio diversification (hedging) in multi-cap investing during the medium-to-long run (short run) only. Like total connectedness, a similar pattern of bilateral connectedness is observed between either of the two indices, thus providing a similar opportunity in the short and long runs. The mid-cap index emerges as the major contributor to total volatility in the system, followed by the small- and large-cap indices, during the analyzed period. The volatility spillover is time-varying in both the time and frequency domains.
\end{abstract}

Keywords: volatility spillover; connectedness; large-, mid-, and small-cap; portfolio diversification and hedging; time; frequency domain

JEL Classification: G11; G12; G14

\section{Introduction}

With the spike in volatility in the Indian stock market towards the middle of 2018, a sharp fall was observed in mid- and small-cap stocks, ${ }^{1}$ while the large-cap stocks managed to withstand the selling pressure as investors sought refuge in the selected large-cap stocks. ${ }^{2}$ Wang et al. (2014), highlighting capitalization as a style of investing in their study, mentions the relative stability (growth potential) of investment in large-cap (mid-and small-cap) stocks. Huang (2007) finds international portfolio diversification opportunities in small-cap stocks because of their low average correlation with their developed market counterparts. Moreover, as far as global pricing is concerned, it is more pronounced in large-cap than small-cap stocks, according to Carrieri et al. (2004), because the pricing of large-cap stocks is associated with their presence in national stock indices, international operations, cross-listing in foreign stock exchanges, and regular tracking by both domestic and international institutional investors. On the other hand, because of the high transaction costs due to a lack of liquidity and the nonavailability of information, small-cap stocks are less accessible to investors (both domestic and international). Therefore, small-cap stocks are priced in terms of local, or idiosyncratic, risk factors, whereas large-cap stocks are driven markedly by common global factors, according to Eun et al. (2008). 
Looking at the annual return spread between mid- and large-cap, and small- and large-cap indices (see Figure 1), there are few peaks and troughs observed. The peaks (troughs) indicate the underperformance (or overperformance) of mid- and small-cap indices vis-à-vis the large-cap index. This relative over- and underperformance by largecap stocks is dynamic, with changes in the financial and economic environment over time. This time-varying return dynamics in different cap stocks is consistent with the business cycle effect, wherein during expansion, small-cap stocks are better performers than their large-cap counters, according to Kim and Burnie (2002) and Switzer (2010). Switzer and Tang (2009) attribute the superior performance of small-cap stocks to fundamental factors, such as better governance due to the close nexus between innovation and entrepreneurship. Moreover, in the long-term horizon, large-cap stocks are outperformed by their small-cap counterparts, argues Siegel (2021). Theoretically, this cross-market connectedness could be attributed to various channels, such as the channel of flight to quality Li (2002), Baur and Lucey (2009), the channel of portfolio rebalancing/diversification Baur and Lucey (2009), and the channel of information transmission Fleming et al. (1998), Underwood (2009). Reinganum (1999) states that return connectedness dynamics are critical for the management of exposure to different cap stocks for portfolio diversification. As far as riskaverse investors are concerned, they are negatively affected by volatility and its spillover, which is the direct translation of risk Wang and Wang (2019). Mensi et al. (2020) mention the impact of volatility and its spillover on portfolio diversification and hedging opportunities between metal and energy commodities.

Since our focus is the modeling of volatility spillover, the portfolio implication of our study is in line with the argument put forward by Bai and Green (2010). According to Bai and Green (2010), lower connectedness/integration between the assets leads to possible diversification. This means that an asset is a potential diversifier if it is least affected by spillover originating from the financial system.

We have used the Diebold and Yilmaz (2012) spillover methodology to estimate the total, net, and pairwise net volatility spillover between large-, mid-, and small-cap stocks in the Indian context. ${ }^{3}$ This was based on the forecasted error variance decomposition (FEVD) of the vector autoregressive framework. Although the spillover of volatility is studied using the GARCH class of models (for example, Beirne et al. 2013; Lin 2013; Li and Giles 2015), it has limited ability to measure the spillover dynamics Baruník et al. (2016a). Nevertheless, the Diebold and Yilmaz (2012) time-domain spillover analysis ignores spillover across different frequencies, which could be asymmetric and attributed to heterogenous market participants in the stock market with distinct term objectives Müller et al. (1997). For example, speculators, traders, and fund managers, and institutional investors and policymakers operate within shorter, medium, and longer time horizons, respectively Mayer (2009) and Baruník et al. (2016a). That is why the study estimates the frequency spillover and connectedness using the Baruník and Krehlik (2018) methodology, which measures the spillover among financial variables that is due to the heterogenous frequency responses of shocks. On the other hand, the Diebold and Yilmaz (2012) timedomain spillover analysis, which is the sum total of the frequency of spillover, is used as a benchmark for comparison. It also helps to understand how much of the total spillover could be attributed to short-, medium- and long-term spillover.

The study addresses the following research questions, which could be instrumental in trading, investing, and managing multi-cap stock portfolios.

First, is there significant volatility spillover and connectedness between large-, mid-, and small-cap stocks? The total connectedness between the large-, mid-, and small-cap stocks is critical for diversification opportunities between all three cap stocks. Second, how are they bilaterally connected and measured by the pairwise net spillover index? This will depend on the degree of diversification between either of two cap stocks (i.e., between large- and mid-, small- and large-, and mid- and small-cap stocks). Third, which market cap is leading or lagging across the entire stock market? This can be measured through the net volatility spillover index. Fourth, is the volatility spillover asymmetrical 
over the frequency and time-varying? Since financial and economic factors are dynamic, the time-varying total, net, and the pairwise net volatility spillover index were estimated reflecting the evolution of connectedness over time and frequency.

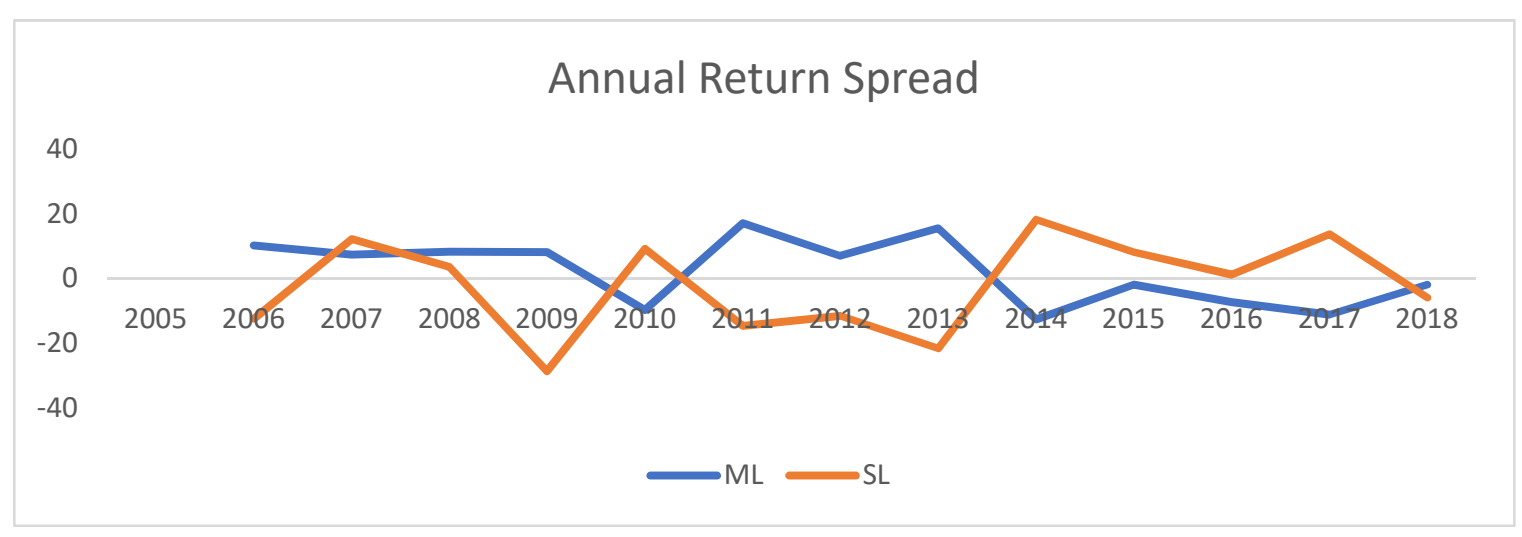

Figure 1. The annual return spread between mid- and large-cap (ML) and small- and large-cap (SL) indices. Source: author's preparation from the sample data of large-, mid-, and small-cap indices taken in the study.

We found that the total volatility spillover between the large-, mid-, and small-cap indices were elevated during the study period. However, the high degree of volatility spillover existed in the short run only, as it declined gradually in the medium-to-long run. That is why the benefit of diversification in the market of cap-based investing can be reaped in the medium-to-long run only. In the system of total volatility spillover, large-cap is the net receiver (transmitter) of volatility from (to) the mid- and small-caps in the short run (medium-to-long run).

The contributions of our paper to the literature are as follows: First, the study is the first of its kind to apply the volatility spillover framework in studying the connectedness dynamics between different market capitalization stocks from the diversification and hedging perspective. Second, the study estimates the volatility spillover in both the time and frequency domains, using the methodology of Diebold and Yilmaz (2012), and Baruník and Krehlik (2018), respectively.

The rest of the paper is organized as follows: Section 2 presents an overview of the literature; Section 3 explains the methodology; Section 4 describes the data; and Section 5 presents the empirical results, followed by discussion; and finally, Section 6 concludes the study.

\section{Literature Review}

The literature review is conducted from two different dimensions. First, from the methodological perspective, where we find a gap in the area of the application of the volatility spillover methodology and, second, from the perspective of equity as an asset class, where it is found that the portfolio opportunities in multi-cap spaces has not been adequately researched.

The following are a few studies found in the area of the application of volatility spillover using the vector autoregressive framework in multi-asset and multi-market contexts related to bonds, stocks, and commodities, etc. Baruník et al. (2016b) applied the methodology of Diebold and Yilmaz (2012) to 21 of the highest capitalization stocks belonging to different sectors. Although they have extended the work of Diebold and Yilmaz (2012) in the direction of understanding asymmetric volatility spillover, the study is confined to the time domain across different sectors.

Zhang and Broadstock (2018) studied how the global commodity markets are connected in the face of the global financial crisis, using the approach of Diebold and Yilmaz $(2009,2012)$. They document a dramatic increase in the connectedness across six broader commodity indices and spot crude oil prices following the crisis. Zhang and Broadstock 
(2018) studied the connectedness between oil shocks and the returns of six stock markets around the world using Diebold and Yilmaz $(2009,2012)$. The results are quite interesting because a limited contribution of the oil shock to the world financial system is found, whereas oil price changes are explained by the financial system. Ferrer et al. (2018) tries to find out the connectedness over time and frequencies using the methodology of Baruník and Krehlik (2018) among clean energy companies, crude oil prices, stock prices of the US, and some key financial variables, such as the VIX, the US Treasury 10-year bond yield, and the treasury yield VIX. The results show the predominance of the high-frequency band (short term) over the low-frequency band (long term) in terms of returns and volatility connectedness among the variables. Moreover, in line with Zhang and Broadstock (2018), no critical role for crude oil prices in explaining stock prices is found.

Tiwari et al. (2018) analyzed the nature of spillover across four global asset classes: currency, credit default swap, sovereign bonds, and stocks over the time and frequency domains, using the methodology of Diebold and Yilmaz (2012) and Baruník and Krehlik (2018). The results show that the high-frequency band dominates the connectedness across the four asset classes. Although the overall stocks and CDS markets are the net transmitters of volatility, it is frequency-dependent. Maghyereh et al. (2019), in an interasset context, analyzed the connectedness between gold and Islamic bonds (Sukuk) and Islamic equity over the frequency domain, using the methodology of Baruník and Krehlik (2018). Their results document portfolio diversification between Islamic assets and gold in the short term because of the low level of the volatility spillover. Thus, it can be inferred that the studies done on volatility transmission are related to interassets in the international and national contexts. Ours is the first study to apply the novel volatility spillover framework of DY and BK in the intraequity market context, where we analyze how different market capitalization stocks, such as large-, mid-, and small-cap stocks are connected. As far as the study of market cap indices are concerned, the literature is scant, as discussed below. Copeland and Copeland (2016) investigated the impact of the VIX on the cross-sectional returns of different cap stocks and found that positive innovation in the VIX is associated with higher large-cap, rather than small-cap, stock returns.

Using return dynamics, and its autocorrelation of different cap stocks, Reinganum (1999) finds that dynamically managed exposure to different capitalization stocks results in better economic gains than a fixed allocation to large- and small-cap stocks. Oberholzer and von Boetticher (2015) study the relationship between the South African Rand and five different cap indices of the Johannesburg Stock Exchange using CCC-GARCH $(1,1)$. Semenov (2015), investing the predictability of the return of both open-to-open and closeto-close prices in a sample of AMEX-, NYSE-, and Nasdaq-listed stocks, found that smallcap returns are highly predictable, compared to their mid- and large-cap counterparts, and that open-to-open returns are more predictable than close-to-close returns. Switzer (2010), examining the relative performance of small-cap stocks vis-a-vis large-cap stocks surrounding economic troughs and peaks, found that small-cap stocks tend to outperform (lag) large-cap stocks during the period subsequent (prior) to economic troughs (economic peaks). Asli (2014) examined the risk and return characteristics of well-known small-cap indices in the US market and found a significant difference in both the absolute and riskadjusted returns in the small-cap index category. Further, Gorman (2003) found significant positive abnormal returns in 165 actively managed small-cap portfolios after considering the transaction costs. Sensoy (2017), investing the systematic liquidity risk by referring it to institutional ownership, reveals that mid- and large-cap firms are more exposed to this new source of liquidity risk than their small-cap counterparts. As far as the Indian markets are concerned, a recent study was done by Kumar and Misra (2018) on the liquidity commonality in 50 mid-cap stocks. They found liquidity commonality in half of the sample stocks across the range of liquidity measures.

Although various dimensions related to performance analysis in terms of risk and return, liquidity, and pricing are addressed in the above-discussed literature, the gap that has emerged is related to the connected dynamics between large-, mid-, and small-cap 
stocks, which is a critical factor for portfolio hedging and diversification across market cap stocks. Therefore, ours is the first study to fill this gap by examining the spillover dynamics between large-, mid- and small-cap stocks using the novel VAR-based spillover model of Diebold and Yilmaz (2012), and Baruník and Krehlik (2018), in the time and frequency domains.

\section{Methodology and Data Specification}

\subsection{Diebold and Yilmaz Model}

In our quest to test the volatility connectedness between large-, mid-, and smallcap stocks, we first adopted the traditional spillover model proposed by Diebold and Yilmaz (2012). Starting from a vector autoregression (VAR) model, Diebold and Yilmaz (DY hereafter) then introduced into the VAR system a variance decomposition function that computes the FEVD, defined as the forecast error variance decomposition, obtained from a generalized vector autoregression framework, in order to investigate the interconnectedness across a multivariate series. The K-variable VAR $(p)$ system is defined as:

$$
y_{t}=c+A_{1} y_{t-1}+A_{2} y_{t-2}+\ldots+A_{p} y_{t-p}+u_{t}
$$

In Equation (1), $y_{t}$ denotes the $K \times 1$ vector of series under examination at time, $t$, while $c$ denotes the constant of $K \times 1$ vector of the series. $A$, in Equation (1), depicts the coefficient of the $K \times K$ dimension matrix, while $u_{t}$, depicts white noise with a (possibly nondiagonal) covariance matrix $\sum$. The simplified form of Equation (1) is shown below:

$$
Y_{t}=C+A Y_{t-1}+U_{t}
$$

In Equation (2) above, $A$ represents a $p K \times p K$ dimension matrix, while $Y, C$, and $U$ symbolize the $p K \times 1$ vectors, defined as shown in Equation (3) below:

$$
Y=\left[\begin{array}{l}
y_{t} \\
y_{t-1} \\
\vdots \\
y_{t-p}
\end{array}\right], C=\left[\begin{array}{l}
c \\
0 \\
\vdots \\
0
\end{array}\right], A=\left[\begin{array}{l}
A_{1} A_{2} \cdots A_{p-1} A_{p} \\
I_{K} 0 \cdots 00 \\
0 I_{K} 00 \vdots \\
\vdots \cdot \cdots \\
00 \cdots I_{K} 0
\end{array}\right], U=\left[\begin{array}{l}
u_{t} \\
0 \\
\vdots \\
0
\end{array}\right]
$$

In estimating the VAR framework, the impulse response function framework is used to explore the magnitude to which a series in the VAR system influences or contributes to variations in other series. The mean square error of the series from the H-step forecast, $y_{t}$, is denoted as:

$$
M S E\left|y_{i . t}(H)\right|=\sum_{j=0}^{H-1} \sum_{k=1}^{k}\left(e_{i}^{\prime} \Theta_{j} e_{k}\right)^{2}
$$

In Equation (4), $e_{i}$ denotes the $i-t h$ column of $I_{K}, \Theta_{j}=\Phi_{j} P$, and $P$ represents the lower triangular matrix. In estimating the lower triangular matrix, denoted as $\mathrm{P}$, we followed the approach of Pesaran and Shin (1998), and Koop et al. (1996), and used the generalized decomposition framework of the variance covariance matrix, $\Omega_{u}=E\left(u_{t} u_{t}^{\prime}\right)$. Furthermore, $\Phi=J A^{j} J^{\prime}$, where $J=\left|I_{K}, 0, \ldots, 0\right|$. Hence, we computed the contribution of the $k$ to $i$ th variable, as shown in Equation (5):

$$
\theta_{i k, H}=\sum_{j=0}^{H-1}\left(e_{i}^{\prime} \Theta_{j} e_{k}\right)^{2} / M S E\left|y_{i, t}(H)\right|
$$


Following Diebold and Yilmaz (2012), the coherency among the variables in the VAR systems is estimated to shorten all series in $\theta(H)$ from 1 to $K$. Coherency is measured as shown below:

$$
C_{H}=\frac{1}{K} \sum_{i j=1}^{K} \theta_{i j}^{H}(i \neq j),
$$

In making sure the overall connectedness index across the series in the system ranges between 0 and 1, we ignored all of the diagonal elements in the system from Equation (6) above. This estimate measures the magnitude to which the system component's role to deviations is prompted by another series, and not the series itself. A situation where the obtained measure of connectedness is zero suggests that the components in the system are independent, signifying no evidence of spillover effects. However, a value of one suggests that the constituents in the VAR system are highly related.

Since the order of series in the VAR system could affect the impulse response or variance decomposition function, we used the estimating techniques of Diebold and Yilmaz (2012) by employing the generalized decomposition framework put forward by Pesaran and Shin (1998), and Koop et al. (1996), to further explore the validity or robustness of our documented results.

$$
\theta_{i K, H}^{g}=\sigma_{i i}^{-1} \sum_{j=0}^{H-1}\left(e_{i}^{\prime} \Phi_{j} \sum_{u} e_{k}\right)^{2} / \operatorname{MSE}\left[y_{i, t}(H)\right] .
$$

\subsection{Barunik and Krehlik Model}

Subsequently, we followed the Baruník and Krehlik (2018) spillover approach to further explore the coherency and spillovers under different frequency levels. The approach of Baruník and Krehlik (2018, BK hereafter) provides comprehensive details on the extent of connectedness at different frequencies, which aids our understanding of the connectedness levels among the variables under examination. Using this approach, we investigated the frequency levels in which the spillover effect was highest, so that market participants with varied investment horizons can ascertain whether to invest in the short run or long run. To achieve this, Baruník and Krehlik (2018) put forward a model that decomposes the novel DY spillover index model at different frequency levels. In particular, the formulation approach of Baruník and Krehlik (2018) uses the spectral formulation of the variance decomposition framework. The approach is described below. Let us assume the frequency response framework is defined as $\Psi\left(e^{-i \omega}\right)=\sum_{h} e^{-i \omega h} \Psi_{h}$, gained from using the Fourier transform of the coefficients $\Psi$, with $i=\sqrt{-1}$

The generalized causality spectrum over different frequency levels, $\omega \in(-\pi, \pi)$, is denoted as shown below:

$$
(f(\omega))_{j, k} \equiv \frac{\sigma_{k k}^{-1} \mid\left(\left.\left(\Psi\left(e^{-i \omega}\right) \sum\right)_{j, k}\right|^{2}\right.}{\left(\Psi\left(e^{-i \omega}\right) \sum \Psi^{\prime}\left(e^{+i \omega}\right)_{j, j}\right.}
$$

where $\Psi\left(e^{-i \omega}\right)=\sum_{h} e^{-i \omega h} \Psi_{h}$, which represents the Fourier transform of the variance decomposition function, $\Psi$ and $(f(\omega))_{j, k}$, connotes the section of the spectrum of the $j$-th series over the frequency, $\omega$, due to shocks within the $k$-th series. Since the denominator contains the spectrum of the $j$-th series over frequency, $\omega$, we referred to Equation (8) as the magnitude within the frequency causality. We obtained the generalized decomposition of variance over different frequency levels, $\omega$, by assigning weight to the function $(f(\omega))_{j, k}$ by the $j$-th series frequency share of the variance. The above discussion on the weighting function is defined as shown in Equation (9):

$$
\Gamma_{j}=\frac{\left(\Psi\left(e^{-i \omega}\right) \sum \Psi^{\prime}\left(e^{+i \omega}\right)\right)_{j, j}}{\frac{1}{2 \pi} \int_{-\pi}^{\pi}\left(\Psi\left(e^{-i \lambda}\right) \sum \Psi^{\prime}\left(e^{+i \lambda}\right)\right)_{j, j} d \lambda}
$$


Equation (9) above documents the $j$-th series power in the VAR system at frequency $\omega$, summing up the frequency levels to a constant estimate of $2 \pi$. It is worth mentioning that, although the Fourier transform of the variance decomposition function is a complex estimate value, the generalized spectrum, which is the squared coefficient of the weighted complex number value, is a real number. The level of frequency bands is set up formally as $d=(a, b): a, b \in(-\pi, \pi), a<b$. We outline, in Equation (10) below, the generalized decomposition in the variance framework in different frequency levels, $d$, as:

$$
\left(\Theta_{d}\right)_{j, k}=\frac{1}{2 \pi} \int_{d}^{\infty} \Gamma_{j}(\omega)(f(\omega))_{j, k} d \omega .
$$

The generalized decomposition in variance is scaled under different frequency levels, $d=(a, b): a, b \in(-\pi, \pi), a<b$, as presented in Equation (11):

$$
\left(\widetilde{\Theta}_{d}\right)_{j, k}=\left(\Theta_{d}\right)_{j, k} / \sum_{k}\left(\Theta_{\infty}\right)_{j, k}
$$

The connectedness within is expressed over the frequency levels, $d$, as:

$$
C_{d}^{W}=100 \cdot\left(1-\frac{\operatorname{Tr}\left\{\widetilde{\Theta}_{d}\right\}}{\sum \widetilde{\Theta}_{d}}\right)
$$

Lastly, we computed the frequency connectedness over the frequency levels, $d$, as:

$$
C_{d}^{F}=100 \cdot\left(\frac{\sum \widetilde{\Theta}_{d}}{\sum \widetilde{\Theta}_{\infty}}-\frac{\operatorname{Tr}\left\{\widetilde{\Theta}_{d}\right\}}{\sum \widetilde{\Theta}_{\infty}}\right)=C_{d}^{W} \frac{\sum \widetilde{\Theta}_{d}}{\sum \widetilde{\Theta}_{\infty}} .
$$

\section{Data Description}

Daily data for Nifty 50 (large-cap), Nifty Midcap 50 (mid-cap) and Nifty Smallcap 50 (small-cap) have been taken from the official website of the National Stock Exchange of India (NSE), starting from 1 April 2005, the date on which the Nifty Smallcap index was launched, 29 June 2018. The large-cap, represented by the NIFTY 50 Index, accounts for about $62.9 \%$ of the total listed stocks' free-float market capitalization of the NSE, as on 31 March 2017. In terms of traded value, the constituents of Nifty 50 account for $43.8 \%$ of the total traded value on the NSE. The mid-cap is represented by the NIFTY Midcap 50 Index. It accounts for $5.8 \%$ of the free-float market capitalization, and $13 \%$ of the traded value of all the stocks listed on the NSE, as on 31 March 2017. Lastly, the small-cap is represented by the NIFTY Smallcap 50 Index, which accounts for about $2 \%$ of the free-float market capitalization, and $6.2 \%$ of the traded value of all the stocks listed on the NSE, as on 31 March 2017. The daily return was calculated as the logarithmic difference between the closing prices at $t$ and $t-1$.

The index closing price and the return are plotted in Figure 2. The return is estimated as the log closing price at time $t$, minus log closing price at $t-1$. It is evident from the graph that, when there is a high level of return fluctuation, the decline in price is observed and the intensity is high for the small-cap index.

The descriptive statistics of the index return are reported through Panel A of Table 1. The highest mean return is observed for the Nifty_50 large-cap, followed by the Nifty_50 small-cap, and the Nifty_50 mid-cap. In terms of risk, measured by the standard deviation of the return, the mid-cap stocks were more volatile, followed by the small-cap and the large-cap stocks. However, in terms of the risk-adjusted return (coefficient of variation), the large-cap provides the highest return per unit of risk, followed by the small-cap and mid-cap indices. It is important to note that all the series are negatively skewed, which means that the negative returns are more frequent than the positive returns. The high kurtosis value implies the existence of price jumps in the cases of all three indices. The 
ADF test shows that all the return series are stationary. Since the series are subject to jumps, we have applied the Zivot and Andrews test of stationarity, which supports the ADF test results.

Panel B reports the unconditional correlation between the three market cap indices. The presence of a high degree of correlation indicates a lack of diversification benefits across the three market cap indices. That means that all three indices are affected by common factors, which are subject to change with the changes in the financial and economic conditions of the economy. In other words, their connectedness is subject to change, which may provide the opportunity for portfolio diversification. Thus, on one hand, the three indices look fundamentally different in terms of operation (global and local), market structure (liquidity), and pricing (global and idiosyncratic factors and tracked by the analyst). On the other hand, they are showing a high level of correlation. Is this high degree of correlation due to the fundamental transitory factors that affect them in the long run (short run)? Thus, it can be inferred that we cannot outrightly reject the portfolio diversification opportunities between the different market cap stocks just by observing the correlation structures. The real dynamics are masked because of the non-normality nature of all the series, the bivariate nonlinear relationships between the series, ${ }^{4}$ and also the existence of breakpoints (see UDMax F-statistics in Table 1) in their bivariate relationship. Therefore, in order to understand this anomaly, we studied the connection between the three indices in time and frequency (long-, medium-, and short-term), in a time-varying framework, from the portfolio investment and diversification perspective.

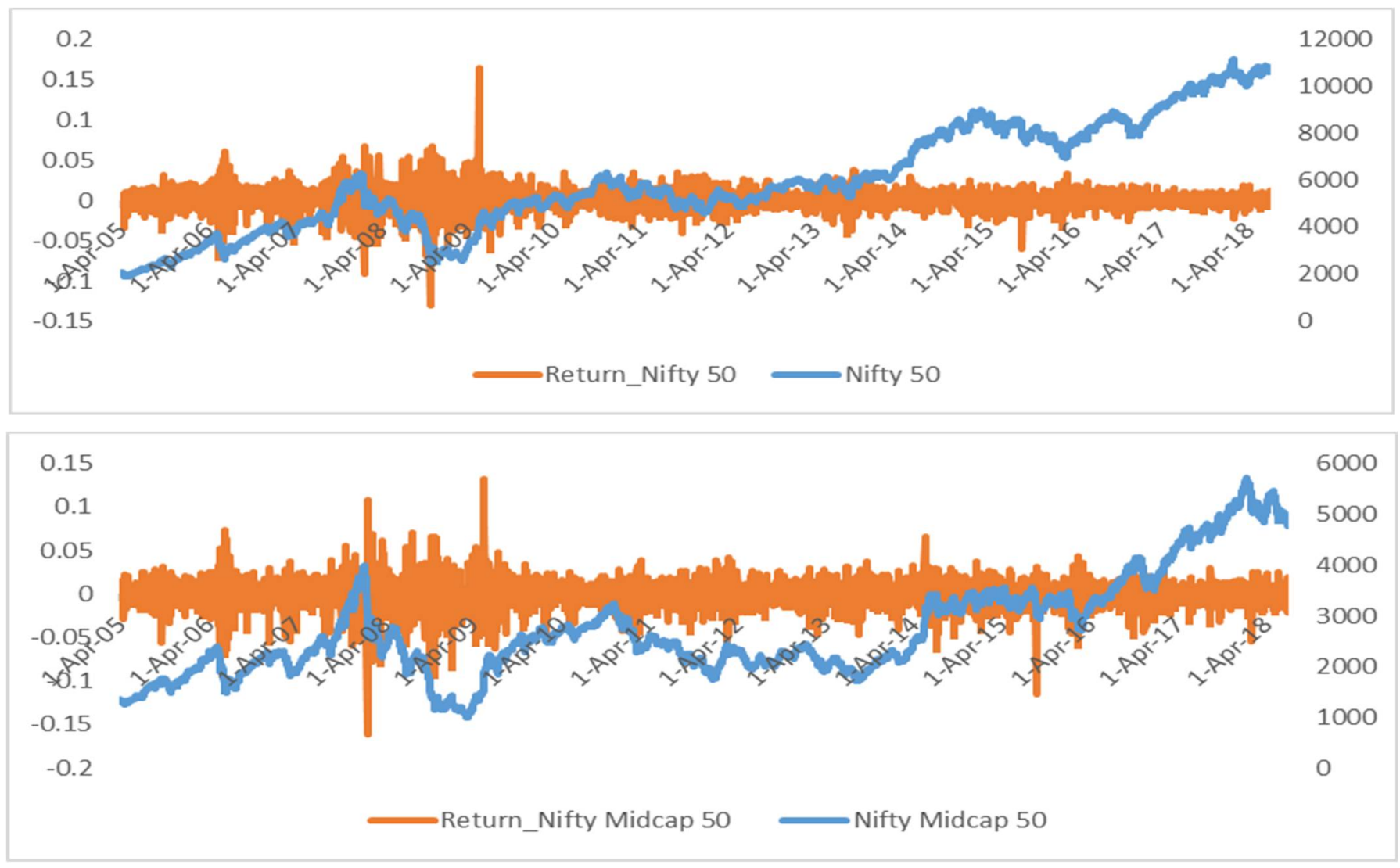

Figure 2. Cont. 


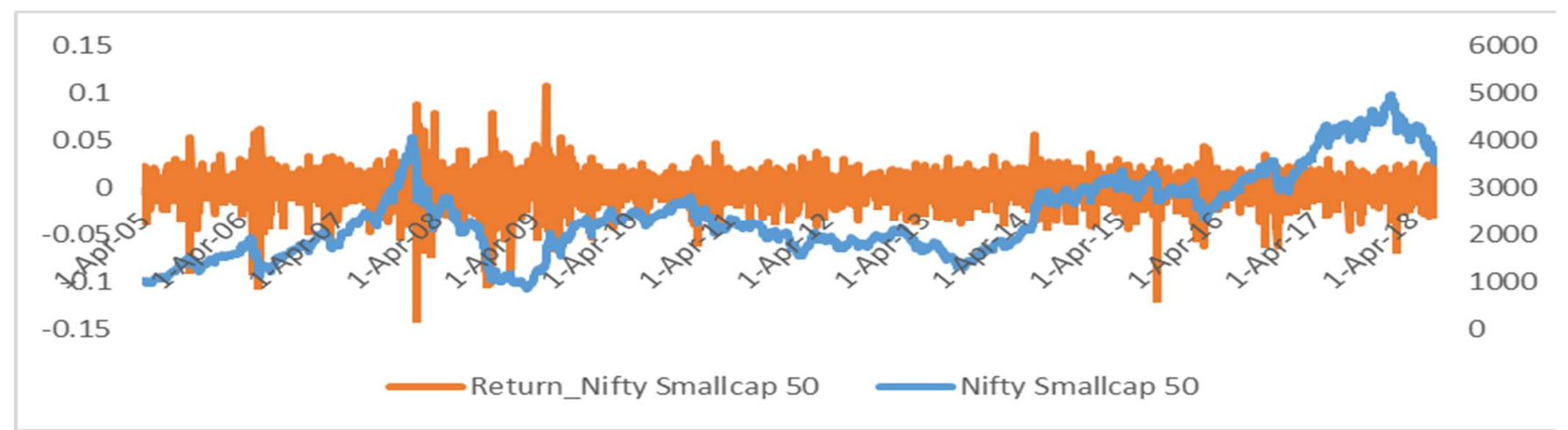

Figure 2. Time series plots of closing prices and returns of large-, mid-, and small-cap indices.

Table 1. Descriptive statistics and correlation matrix of large-, mid-, and small-cap indices daily returns.

\begin{tabular}{|c|c|c|c|}
\hline & $\begin{array}{c}\text { Nifty.50 } \\
\text { (Large-Cap) }\end{array}$ & $\begin{array}{l}\text { Nifty.Midcap.50 } \\
\text { (Mid-Cap) }\end{array}$ & $\begin{array}{c}\text { Nifty.Smallcap.50 } \\
\text { (Small-Cap) }\end{array}$ \\
\hline \multicolumn{4}{|c|}{ Panel A: descriptive statistics } \\
\hline Mean & 0.001 & 0.000 & 0.000 \\
\hline Median & 0.001 & 0.002 & 0.002 \\
\hline Maximum & 0.163 & 0.131 & 0.107 \\
\hline Minimum & -0.130 & -0.162 & -0.138 \\
\hline Std. Dev. & 0.014 & 0.017 & 0.017 \\
\hline Skewness & -0.051 & -0.725 & -0.965 \\
\hline Kurtosis & 13.523 & 10.674 & 9.953 \\
\hline Jarque-Bera & $15,172.320$ & 8358.190 & 7135.885 \\
\hline Probability & $0.000 * * *$ & $0.000 * * *$ & $0.000 * * *$ \\
\hline Observations & 3288 & 3288 & 3288 \\
\hline ADF t-statistic & $\begin{array}{l}-53.937 \\
\left(0.000^{* * *}\right)\end{array}$ & $\begin{array}{l}-50.372 \\
\left(0.000^{* * *}\right)\end{array}$ & $\begin{array}{l}-48.014 \\
\left(0.000^{* * *}\right)\end{array}$ \\
\hline Zivot-Andrews t-statistic & $\begin{array}{l}-54.032 \\
\left(0.011^{* *}\right)\end{array}$ & $\begin{array}{l}-28.629 \\
\left(0.002^{* * *}\right)\end{array}$ & $\begin{array}{l}-27.113 \\
\left(0.001^{* * *}\right)\end{array}$ \\
\hline UDMax F statistic & $\begin{array}{l}\text { Large-mid } \\
106.13^{* *}\end{array}$ & $\begin{array}{l}\text { Large-small } \\
92.79 * *\end{array}$ & $\begin{array}{l}\text { Mid-small } \\
48.02 * *\end{array}$ \\
\hline \multicolumn{4}{|c|}{ Panel B: Unconditional correlation matrix } \\
\hline Large-cap & 1 & & \\
\hline Mid-cap & $\begin{array}{l}0.847 \\
\left(91.258^{* * *}\right)\end{array}$ & 1 & \\
\hline Small-cap & $\begin{array}{l}0.785 \\
\left(72.610^{* * *}\right)\end{array}$ & $\begin{array}{l}0.917 \\
\left(131.795^{* * *}\right)\end{array}$ & 1 \\
\hline
\end{tabular}

*** and ${ }^{* *}$ refer to $1 \%$ and $5 \%$ levels of significance, respectively. UDMax F statistics critical value is 11.7. It denotes 1 to $\mathrm{M}$ globally determined breaks, as per the Bai and Perron (2003) multiple break points test.

\section{Empirical Results and Discussions}

The empirical results are discussed in two parts. The first part is a static analysis through tabular presentation in Section 5.1, and the second part is a dynamic analysis, in a time-varying framework, through graphical presentation in Section 5.2. 


\subsection{Static Analysis of Volatility Spillover}

In this section, the static analysis of the volatility spillover between large-, mid-, and small-cap stocks are presented in the three parts as the total, net, and pairwise net volatility spillover.

\subsubsection{Total Volatility Spillover}

The methodology explained in Section 3 is based on a three-variable VAR that is estimated using an automatically chosen lag length by the Akaike information criterion (AIC). Next, the connectedness between the three market cap indices was estimated, and presented through Table 2, using variance decomposition forecasted for a forecasting horizon $(\mathrm{H})$ of a 100-day period ahead. The forecasting horizon of $\mathrm{H}=100$ days was selected based on the minimum number of days required to estimate the volatility spillover, based on Baruník and Krehlik (2018). The estimated results of the volatility spillover, based on the methodologies of Diebold and Yilmaz (2012) and Baruník and Krehlik (2018), are presented in Table 2.

The total DY connectedness between the large-, mid-, and small-cap indices was $59.93 \%$, which indicates that these three market cap stocks are closely interconnected with each other. That means that around $60 \%$ of the volatility in the system is due to their connectedness, which is in line with the estimated value of the high unconditional correlation and could be attributed to common factors. Thus, this is related to the theoretical connectedness due to the channel-of-information transmission (Fleming et al. 1998; Underwood 2009). Moreover, like the study of Baruník et al. (2016a), in the intersectoral equity market, the high level of connectedness in the inter-capitalization stock market is reported by our study. Furthermore, the results reveal that the mid-cap index contributes most to the system $(20.50 \%)$, followed by the small-cap $(20.11 \%)$, and the large-cap $(19.32 \%)$. It is worth noting here that the difference in the contribution of each index to the volatility spillover is marginal. Although such a high level of connectedness across the market cap indices reduces the diversification opportunity, it increases the hedging opportunity between large-, mid-, and small-cap stocks. However, now the question arises, does this high level of connectedness persist over different timescales? We decomposed the total spillover, frequency-wise, using the Baruník and Krehlik (2018) method, in high- (i.e., Freq. 1, which corresponds to 1 to 4 days), medium- (i.e., Freq. 2, which corresponds to 4 to 10 days), and low- (i.e., Freq. 3, which corresponds to more than 10 days) frequencies. We found that the degree of connectedness implied by the total volatility spillover index gradually declined from $41 \%$ to $12.07 \%$, and finally to $6.86 \%$, in short-, medium-, and long-term indices, respectively. This is in line with the findings of Ferrer et al. (2018), in the case of energy companies, and Tiwari et al. (2018), in the cases of interasset classes. However, the contribution of each market cap index to the total spillover varied with the change in the frequency. For example, in the short-term period, i.e., at Freq. 1, the contribution of the large-cap is highest, followed by the mid-cap and small-cap. However, in the medium term, i.e., at Freq. 2, the contribution of the small-cap is the highest, followed by the medium-cap and large-cap. In the long period, i.e., at Freq. 3, the contribution from the small-cap is the largest, followed by the mid-cap and the large-cap. Thus, based on the frequency domain results, we could deduce that portfolio diversification opportunities increase moving from the short to the long run, and it is highest in the long run. On the other hand, the high level of connectedness in the short run offers hedging opportunities across different capitalization stocks, which gradually declines in the long run. The implication of the results for a portfolio manager is that large-cap stocks can be used for hedging and portfolio diversification purposes in the short and long runs, respectively, by small- and mid-cap investors. Although, in the short run, the diversification opportunities disappear because of the high level of spillover, it provides a trading opportunity, in general, and a volatility-timed trading strategy, in particular, due to the risk-rerun tradeoff mismatch in the short run. This confirms the findings of Moreira and Muir (2017), where timing the 
volatility through trading brings an increased utility to mean-variance investors, and even also for long-term investors, according to Moreira and Muir (2019).

Going deeper into the connectedness, it is evident that $68 \%$ of the total connectedness in the system is due to short-term factors, such as euphoria and pessimism, and that $11 \%$ is due to long-term fundamental factors, which confirms the findings of Elias (2016). In such a system, the volatility spillover acts as a proxy for information spillover directed from the mid- and small-cap indices to the large-cap indices. However, in the medium-to-long term, the scenario gets reversed and information spills over from large-cap to mid-cap and small-cap indices. This has implications for the pricing of different cap stocks, according to Eun et al. (2008). In their study, Eun et al. (2008) mention that large-cap stocks are driven by global factors, and small-caps are driven by local or idiosyncratic factors.

Table 2. Volatility Spillover between large, mid, and small-cap indices.

\begin{tabular}{ccccc}
\hline \multicolumn{1}{c}{ Panel A: Diebold and Yilmaz (2012) volatility spillover matrix between Nifty.50 (Large-cap), Nifty. Midcap.50 (Mid-cap), and } \\
Nifty.Smallcap.50 (Small-cap) indices
\end{tabular}

Panel B: Baruník and Krehlik (2018) volatility spillover matrix between Nifty.50 (Large-cap), Nifty. Midcap.50 (Mid-cap), and Nifty.Smallcap.50 (Small-cap) indices

\begin{tabular}{|c|c|c|c|c|c|}
\hline \multicolumn{6}{|c|}{ Freq1: 3.14 to 0.79 (corresponds to 1 to 4 days) } \\
\hline & Nifty.50 & Nifty.Midcap.50 & Nifty.Smallcap.50 & FROM_ABS & FROM_WTH \\
\hline Nifty.50 & 30.93 & 23.06 & 20.38 & 14.48 & 21.06 \\
\hline Nifty.Midcap.50 & 18.64 & 26.48 & 22.41 & 13.68 & 19.9 \\
\hline Nifty.Smallcap.50 & 16.48 & 22.03 & 25.83 & 12.84 & 18.67 \\
\hline TO_ABS & 11.71 & 15.03 & 14.26 & 41 & \\
\hline TO_WTH & 17.03 & 21.86 & 20.75 & & \\
\hline \multicolumn{6}{|c|}{ Freq2: 0.79 to 0.31 (corresponds to 4 to 10 days) } \\
\hline & Nifty.50 & Nifty.Midcap.50 & Nifty.Smallcap.50 & FROM_ABS & FROM_WTH \\
\hline Nifty.50 & 7.4 & 5.11 & 4.52 & 3.21 & 16.08 \\
\hline Nifty.Midcap.50 & 6.29 & 7.81 & 6.7 & 4.33 & 21.67 \\
\hline Nifty.Smallcap.50 & 6.02 & 7.55 & 8.53 & 4.53 & 22.65 \\
\hline TO_ABS & 4.1 & 4.22 & 3.74 & 12.07 & \\
\hline TO_WTH & 20.54 & 21.14 & 18.72 & & \\
\hline \multicolumn{6}{|c|}{ Freq3: 0.31 to 0.0 (corresponds to more than 10 days) } \\
\hline & Nifty.50 & Nifty.Midcap.50 & Nifty.Smallcap.50 & FROM_ABS & FROM_WTH \\
\hline Nifty.50 & 3.71 & 2.44 & 2.44 & 1.63 & 14.44 \\
\hline Nifty.Midcap.50 & 3.58 & 4.19 & 3.89 & 2.49 & 22.1 \\
\hline Nifty.Smallcap.50 & 3.76 & 4.47 & 5.33 & 2.74 & 24.35 \\
\hline TO_ABS & 2.45 & 2.31 & 2.11 & 6.86 & \\
\hline TO_WTH & 21.72 & 20.46 & 18.72 & & \\
\hline
\end{tabular}

Note: Rows and columns represent volatility spillover to and from the markets, respectively. Numbers in bold indicate total spillover index, i.e., systemwide spillover. The diagonal (off-diagonal) values indicate spillovers from own market (other markets). 


\subsubsection{Net Volatility Spillover}

The results of the net directional connectedness between large-, mid-, and small-cap indices are presented in Table 3. As is evident from the Diebold and Yilmaz (2012) results, in the case of the large-cap, the net volatility spillover is negative, while in the cases of the midand small-cap indices, it is positive. That means that large-cap (mid- and small-cap) stocks are the average receivers (transmitters) of volatility from (to) the system. In the frequency domain, BK spillover shows that, at Freq. 1, the results are in line with the Diebold and Yilmaz (2012) estimates, i.e., the large-cap has a net negative spillover, while the mid- and small-cap indices have net positive spillover. That means that short-term or transitory factors dominate the average volatility spillover dynamics of different cap stocks. However, at Freq. 2 and Freq. 3, the nature of the spillover is reversed, i.e., the large-cap has net positive spillover, whereas the mid-cap and small-cap indices have net negative spillover, with the small-cap being the highest recipient of volatility in the system. Thus, the sign of net spillovers between the indices changes at relatively longer horizons. The implication of the net volatility spillover is that, in order to control volatility in the market, policy measures should be directed to the mid-and small-cap indices. If only the time-domain approach was followed, policymakers would have directed policy measures to reduce volatility in the mid-cap index only. Thus, frequency-dependent policy measures could be more effective in controlling the stock market volatility. This also has implications for index investors investing for different time periods. For example, for short-term investors in the large-cap index, in addition to its own volatility, it is pertinent to understand how much volatility spills over from the small-and mid-cap indices, and vice versa, in the long run.

Table 3. Net volatility spillover matrix between Nifty.50 (large-cap), Nifty. Midcap.50 (mid-cap), and Nifty.Smallcap.50 (small-cap) indices.

\begin{tabular}{llll}
\hline & Nifty.50 & Nifty.Midcap.50 & Nifty.Smallcap.50 \\
\hline Panel A: Overall Diebold and Yilmaz (2012) & -1.06 & 1.05 & 0.01 \\
\hline Panel B: Frequency domain Baruník and Krehlik (2018) & & & 1.43 \\
\hline Freq. 1 & -2.77 & 1.35 & -0.79 \\
\hline Freq. 2 & 0.89 & -0.11 & -0.63 \\
\hline Freq. 3 & 0.82 & -0.19 & \\
\hline
\end{tabular}

Note: The net volatility spillover measured as volatility spillover to the system (i.e., other market) minus (-) volatility spillover from the system.

\subsubsection{Pairwise Net Volatility Spillover}

While overall and net volatility spillover, and their dynamics over different timescales, have implications for the construction and management of multi-cap portfolios, the pairwise volatility spillover, and its dynamics over different timescales, could be one of the important inputs for managing portfolios consisting of either of the two indices, or stocks belonging to either of the two indices.

The results of the pairwise net directional connectedness in the time and frequency domains are reported in Table 4. As is evident from the DY (2012) results, the average pairwise net directional connectedness is negative for large- and mid-cap, and large- and small-cap pairs, while it is positive for the crossover pair, i.e., the mid- and small-caps. That means that large-cap stocks are the receiver of volatility from mid- and small-cap stocks, while between mid- and small-cap stocks, it is the latter that is the receiver of volatility from the former. At Freq. 1, i.e., in the short term, the average position of the large-cap index, as a net receiver of volatility, is not changed in cases of the large-mid- and large-small-cap pairs, while in the case of the mid-small-cap pair, the mid-cap becomes the net receiver of the volatility in the short term. That means that the average volatility in the large-mid-, and large-small-cap pairs can be attributed to short-term or transitory factors. In the case of the medium to long terms, at Freq. 2 and Freq. 3, the sign of connectedness is 
changed from negative to positive for the first two pairs, whereas it is very volatile across different frequencies in the case of the mid-small-cap pair. That means that the large-cap becomes the net transmitter of volatility, in the medium to long term, to mid- and small-cap stocks. The total pairwise connectedness, which measures the pairwise dependency, is the highest between the mid- and small-caps (67.06), followed by the large- and mid-cap (59.13), and large- and small-cap (53.6). ${ }^{5}$ This high-level average dependency is negative for portfolio diversification between any two cap stocks, but they can act as a hedges for each other. However, as we move from the short to medium and, finally, to the long term, the pairwise total connectedness is reduced across all three pairs, thereby providing portfolio diversification opportunities. These results again highlight the importance of connectedness at different frequencies between these market cap indices.

Table 4. Pairwise net volatility spillover matrix between Nifty.50 (large-cap), Nifty. Midcap.50 (mid-cap), and Nifty.Smallcap.50 (small-cap) indices.

\begin{tabular}{ccccccc}
\hline & & Pairwise & & \multicolumn{2}{c}{ Pairwise Total } \\
\hline & Large-Mid & Large-Small & Mid-Small & Large-Mid & Large-Small & Mid-Small \\
\hline Overall DY (2012) & -2.11 & -1.08 & 1.06 & 59.13 & 53.6 & 67.06 \\
\hline Frequency domain BK (2018) & & & & & & \\
\hline Freq. 1 & -4.42 & -3.9 & -0.38 & 41.7 & 36.86 & 10.54 \\
\hline Freq. 2 & 1.18 & 1.5 & 0.85 & 11.4 & 14.25 \\
\hline Freq. 3 & 1.14 & 1.32 & 0.58 & 6.02 & 6.2 & 8.36 \\
\hline
\end{tabular}

Note: The pairwise net volatility spillover measured as volatility spillover to another market minus (-) volatility spillover from another market.

\subsection{Dynamic Volatility of Spillover Analysis}

In this section, a rolling-windows analysis of the volatility spillover between large-, mid-, and small-cap stocks are presented in the three parts as the total, net, and pairwise time-varying volatility spillover. For this purpose, a 250-day rolling window is used with a forecast horizon of 100 days.

\subsubsection{Rolling Total Volatility Spillover}

Although a snapshot of the average volatility spillover can be inferred from the static analysis, this misses the cyclical and secular spillover. This is important, as the evolution of the market during the sample period had been through the period of turbulence caused by the global financial crisis (GFC), the European banking crisis, and the period of tranquility following the crisis. Therefore, in this section, we construct the time-varying volatility spillover index at the time and frequency domains to investigate the secular and cyclical aspects of the volatility spillover between large-, mid-, and small-cap stocks.

The Diebold and Yilmaz (2012) overall, and the Baruník and Krehlik (2018) frequencywise time-varying volatility spillover index, are presented in Figure 3.

The total connectedness between the three indices was the highest during the financial crisis, and it remained at the top up until 2012 (2011-2012 marks the start of the European crisis). This is in line with the finding of Zhang and Broadstock (2018), who mention the high-level connectedness between the commodities markets at the time of the crisis. That means that both short-term transitory and long-term fundamental factors were equally affected during the period crisis. The period of a high level of connectedness is followed by a phase where the volatility spillover declined to its lowest level of around 50 during 2013-2015. This phase marks the formation of a new government at the center with an ablute majority, ${ }^{6}$ which was expected to drive the much-needed financial and economic reforms. The third phase of spillover started from 2015 to 2017, when the spillover index returned to the previous high level. During this phase, the Indian stock market in general was affected by the onslaught of the nonperforming asset crisis, which paralyzed the entire 
banking system. After that, the overall connectedness between the indices started declining through 2018, where the stock market again made another historical high against the backdrop of the expected reforms (such as the introduction of GST, and the enactment of the IBC act) undertaken by the government, which boosted the confidence of the investors. Moreover, the overall pattern of connectedness is similar to the $\mathrm{f}$ short-term connectedness between the three indices. Thus, the dominance of the short-term factors in the average volatility spillover between all three cap markets was reinforced. That means that the overall connectedness over time between the indices is dominated by the short-term transitory, and not the fundamental, factors affecting the indices. In the medium and long terms, the connectedness looks similar, and the highest values of the index are reported as 16 and 10, respectively. Thus, the lowest time-varying connectedness in the medium to long term periods further strengthens the existence of multi-cap portfolio diversification opportunities.

Overall spillovers

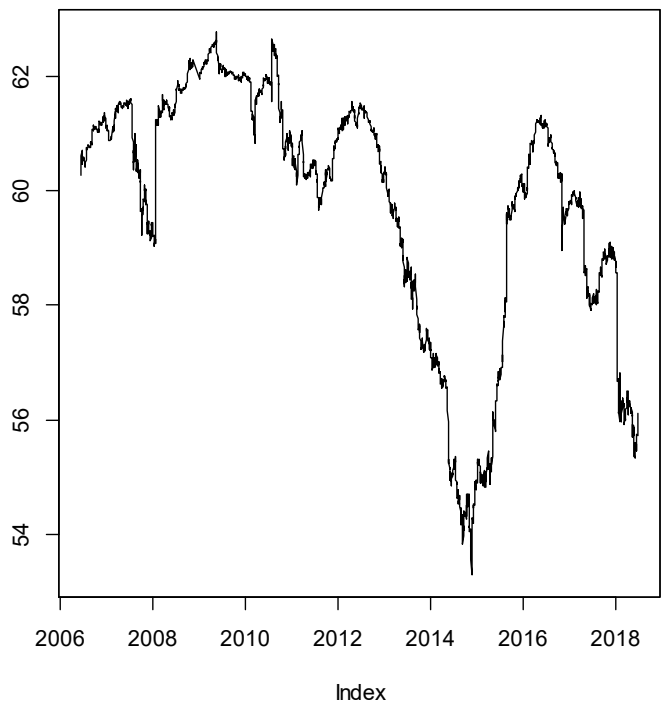

Overall spillovers on band: 0.79 to 0.31

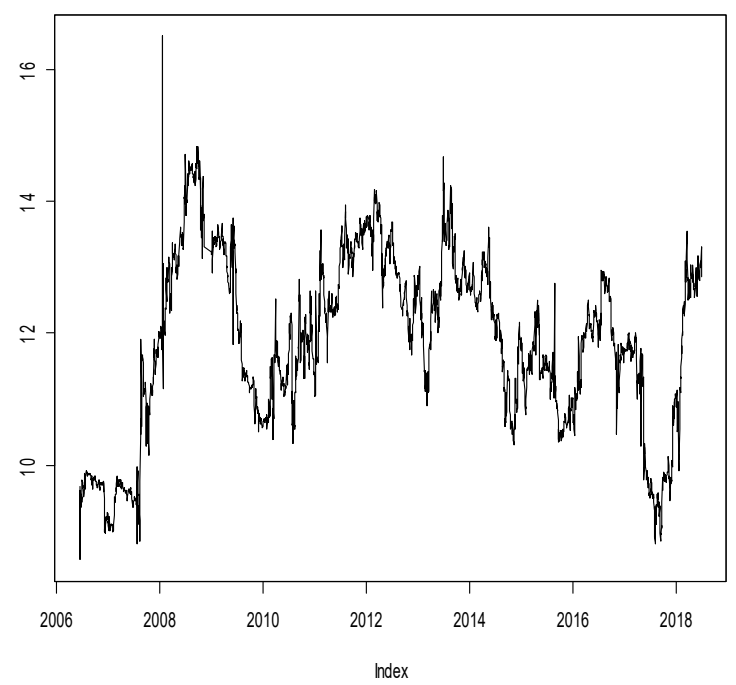

Overall spillovers on band: 3.14 to 0.79

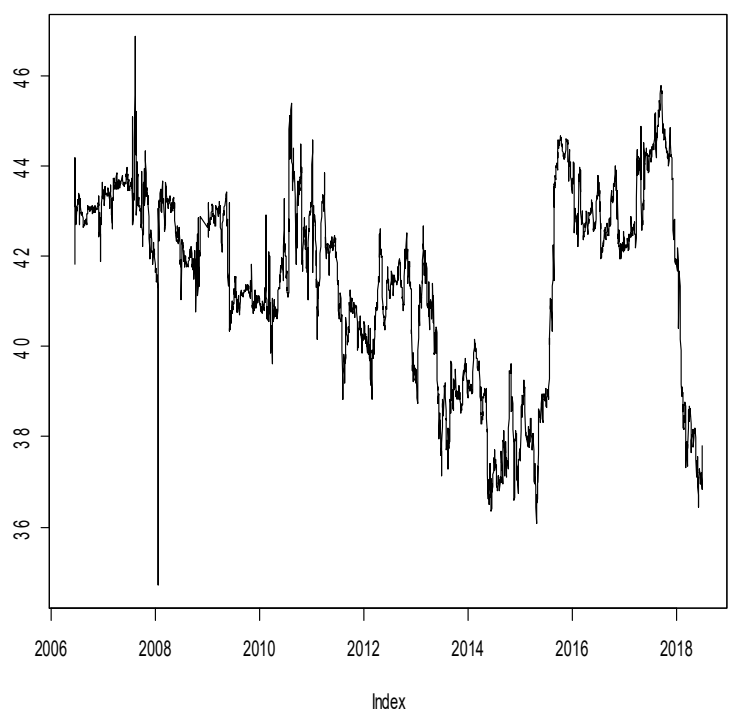

Overall spillovers on band: 0.31 to 0.00 .

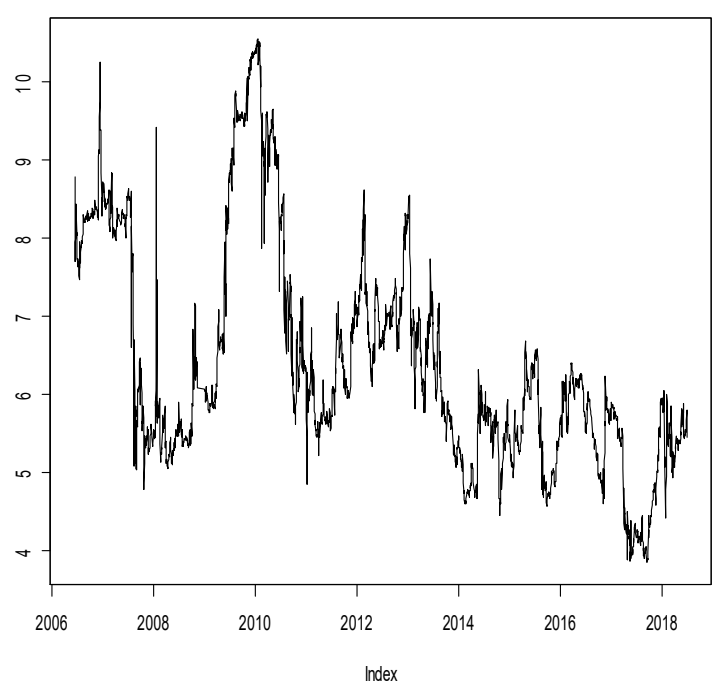

Figure 3. Diebold and Yilmaz (2012) total volatility spillover index presented through the top left figure (i.e., overall spillover) followed by the Baruník and Krehlik (2018) total volatility spillover at frequency bands of 3.14-0.79, 0.79-0.31, and $0.31-0.00$, for a time period of 1-4 (short-term), 4-10 (medium-term), and more than 10 days (long-term) respectively. 


\subsubsection{Rolling Net Volatility Spillover}

To understand what happens to the net position of each index in the system, as a transmitter or receiver of volatility, over a period of time, the estimated rolling net directional spillover index (i.e., the difference between the gross directional volatility spillover to others and from others in the system for each index) is presented in Figure 4. In Appendix A, we have presented the rolling gross directional volatility from each index (Figure A1), and to each index (Figure A2), in the system. It is evident that, the large-cap index is the net receiver and the mid- and small-cap indices are the net transmitters of volatility, and the degree of connectedness increases over the period. In addition, if we look at the connectedness over the short term (Freq. 1), it resembles the overall connectedness over time. Moreover, this net connectedness dynamics gets reversed in the medium and long terms, where the large-cap index becomes the net transmitter, and the mid- and small-cap indices are the net receivers of volatility in the system over the sample period. Thus, the volatility in the system is due to short-term factors coming from the mid-and small-caps, probably because, during the volatile period in the short term, investors are shifting their portfolio from mid- and small-cap stocks to large-cap stocks because large-cap stocks are relatively stable, according to Wang et al. (2014).

\subsubsection{Rolling Pairwise Net Volatility Spillover}

In this section, we look at how the bilateral connectedness between any two indices evolves over a period. The rolling pairwise net directional volatility spillover index in the time and frequency domains are presented in Figure 5. The pairwise net directional spillover between either of two indices is highly volatile. Although it is mostly positive, sometime around 2010 and 2015, big negative swings in the net connectedness, especially for the mid-cap and small-cap indices, are observed. The dynamics of the factors contributing to the average pairwise net directional connectedness of each pair changes across time and frequency. The impact of these dynamics can be linked to the annual return spread, presented in Figure 1. For example, when starting from 2017 through 2018, the mid-small cap pairwise net connectedness gets stabilized, and the highest annual return spread is observed between them. With regard to the frequency domain pairwise net volatility spillovers, not much change in the pairwise net connectedness is observed in comparison to the overall pairwise connectedness between either of the two market cap indices. 

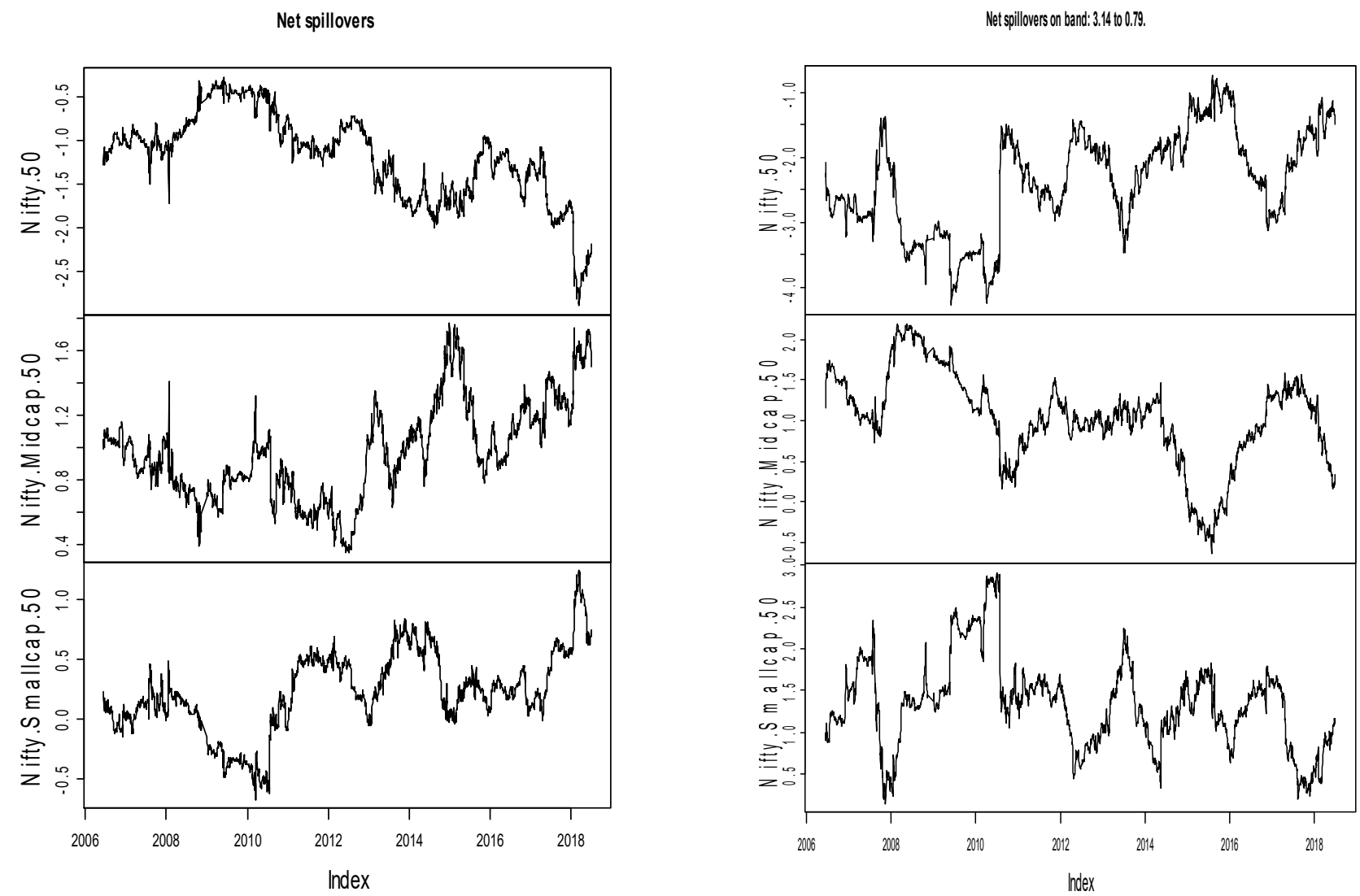

Net spillovers on band: 0.79 to 0.31

Net spillovers on band: 0.31 to 0.00
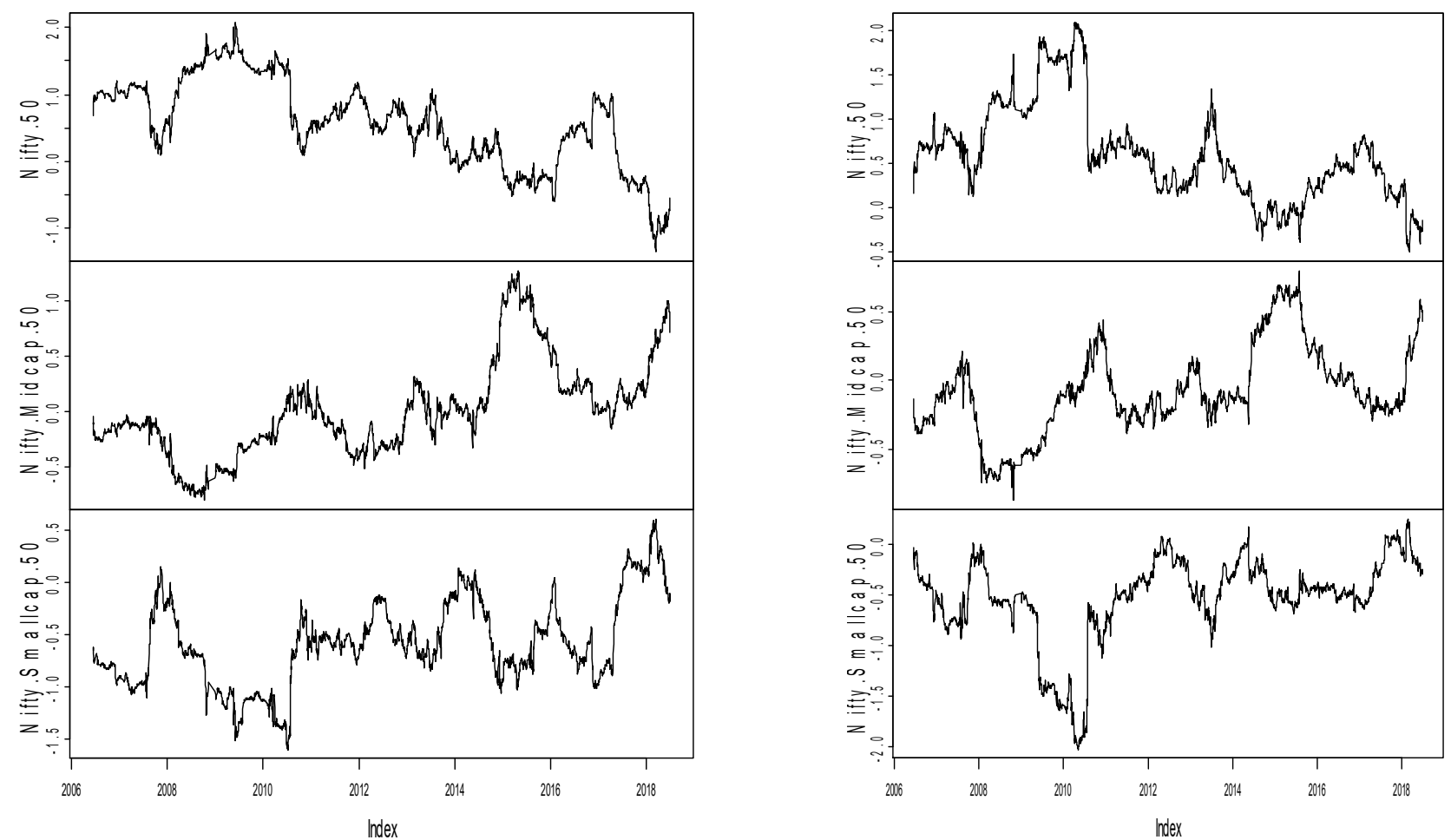

Figure 4. Diebold and Yilmaz (2012) net volatility spillover index presented through the top left figure (i.e., net spillovers) followed by Baruník and Krehlik (2018) total volatility spillover at frequency bands of 3.14-0.79, 0.79-0.31, and 0.31-0.00, for a time period of 1-4 (short-term), 4-10 (medium-term), and more than 10 days (long-term), respectively. 
Painwise spillovers
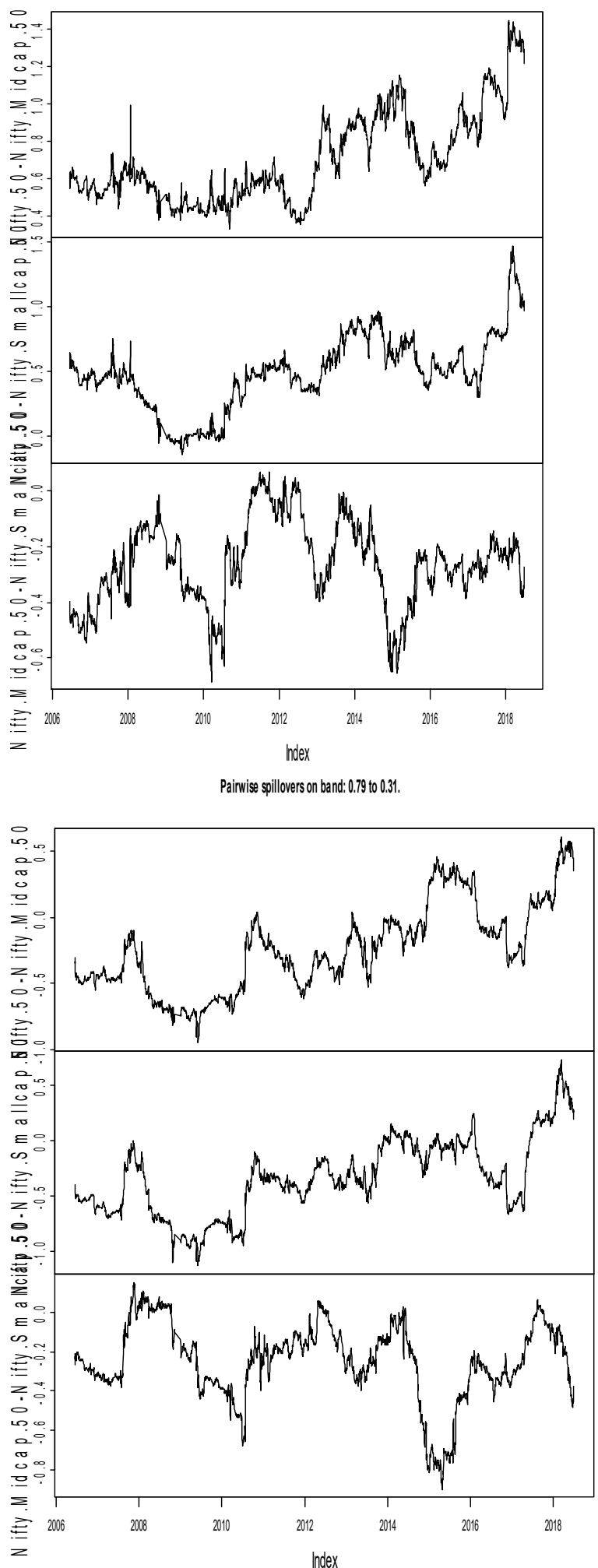

Pairwise spillovers on band: 3.14 to 0.79 .

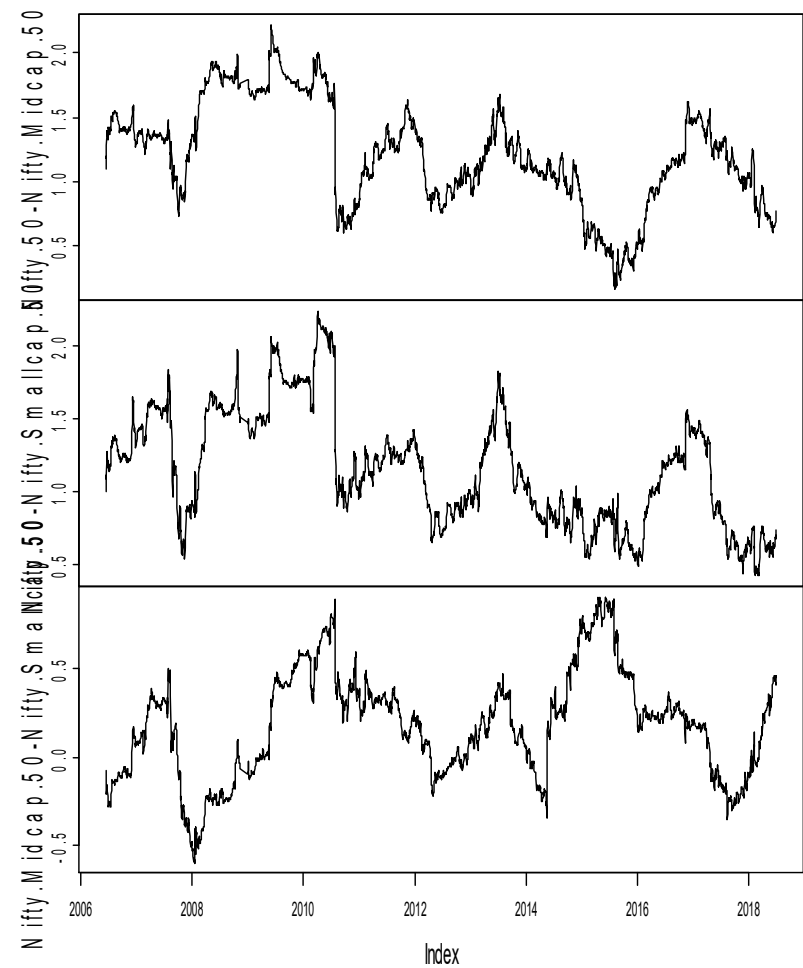

Painvise spillovers on band: 0.31 t 0 0.00

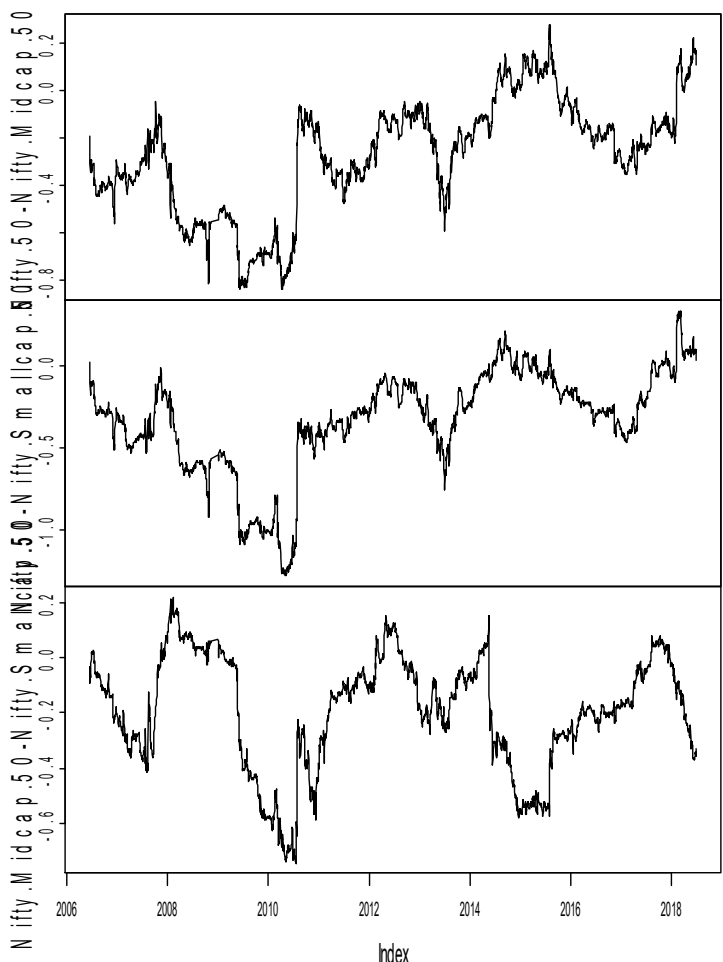

Figure 5. Diebold and Yilmaz (2012) pairwise net volatility spillover index presented through the top left figure (i.e., net spillovers), followed by the Baruník and Krehlik (2018) total volatility spillover at frequency bands of 3.14-0.79, 0.79-0.31, and $0.31-0.00$, for a time period of 1-4 (short-term), 4-10 (medium-term), and more than 10 days (long-term), respectively. 


\section{Conclusions}

In the market cap-based style of investment, the stocks are classified as large-, mid-, and small-cap stocks. Although, unconditionally, these indices are highly correlated, they are distinct in terms of risk, return, and other parameters, such as liquidity and pricing. This study examines, in an intraequity market context, the connected dynamics between these three indices using the volatility spillover index frameworks of Diebold and Yilmaz (2012) and Baruník and Krehlik (2018), in the time and frequency domains, respectively. The total spillover index was found to be $59.93 \%$, which indicates a high level of connectedness between all three market cap indices. However, the degree of total connectedness declined from $41 \%$ to $12.07 \%$, and finally to $6.86 \%$, as we moved from the short-to-medium to the long term during the study period. Thus, this provides opportunities for portfolio diversification and hedging in the market-cap style of investing over the long run and short run, respectively. Therefore, in the volatile equity market, investors can use the large-cap stocks for the purpose of hedging in the short run. Similar to the total connectedness, a similar pattern of bilateral connectedness was observed between either of the two indices, thus providing hedging and diversification opportunities in the short and long runs, respectively, between the large-mid, large-small, and mid- and small-cap indices. In this high level of systematic connectedness, the mid-cap index is the major contributor to volatility, followed by the small- and the large-cap indices, during the analyzed period. Thus, policy measures to contain volatility in the market should be directed towards the appropriative capitalization stocks that transmit volatility to the system at different frequencies. The stability of the magnitude and direction of this connectedness is observed in both the time and frequency domains from the time-varying evolution of the volatility spillover.

Author Contributions: Conceptualization and writing—original draft preparation, S.K.J.; methodology, software, validation, and formal analysis A.K.T.; writing—review, A.D.; methodology, E.J.A.A. All authors have read and agreed to the published version of the manuscript.

Funding: This research received no external funding.

Data Availability Statement: Data available on request due to restrictions e.g., privacy or ethical.

Conflicts of Interest: The authors declare no conflict of interest.

\section{Appendix A}

Rolling gross directional volatility spillover from (Figure A1), and to (Figure A2), of each of the indices in the system in the time and frequency domains. 

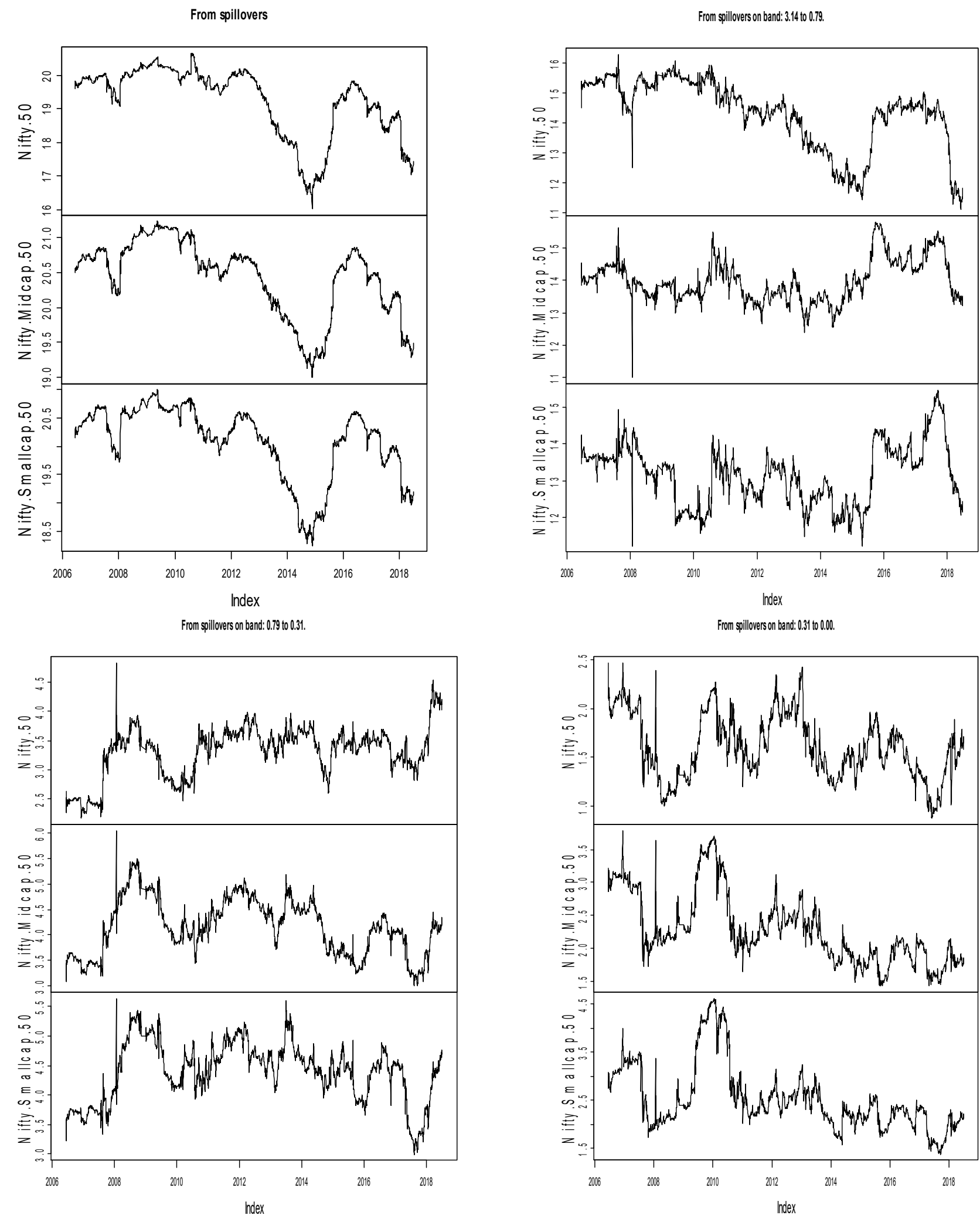

Figure A1. Diebold and Yilmaz (2012) and Baruník and Krehlik (2018) rolling gross volatility spillover from others to the system. 

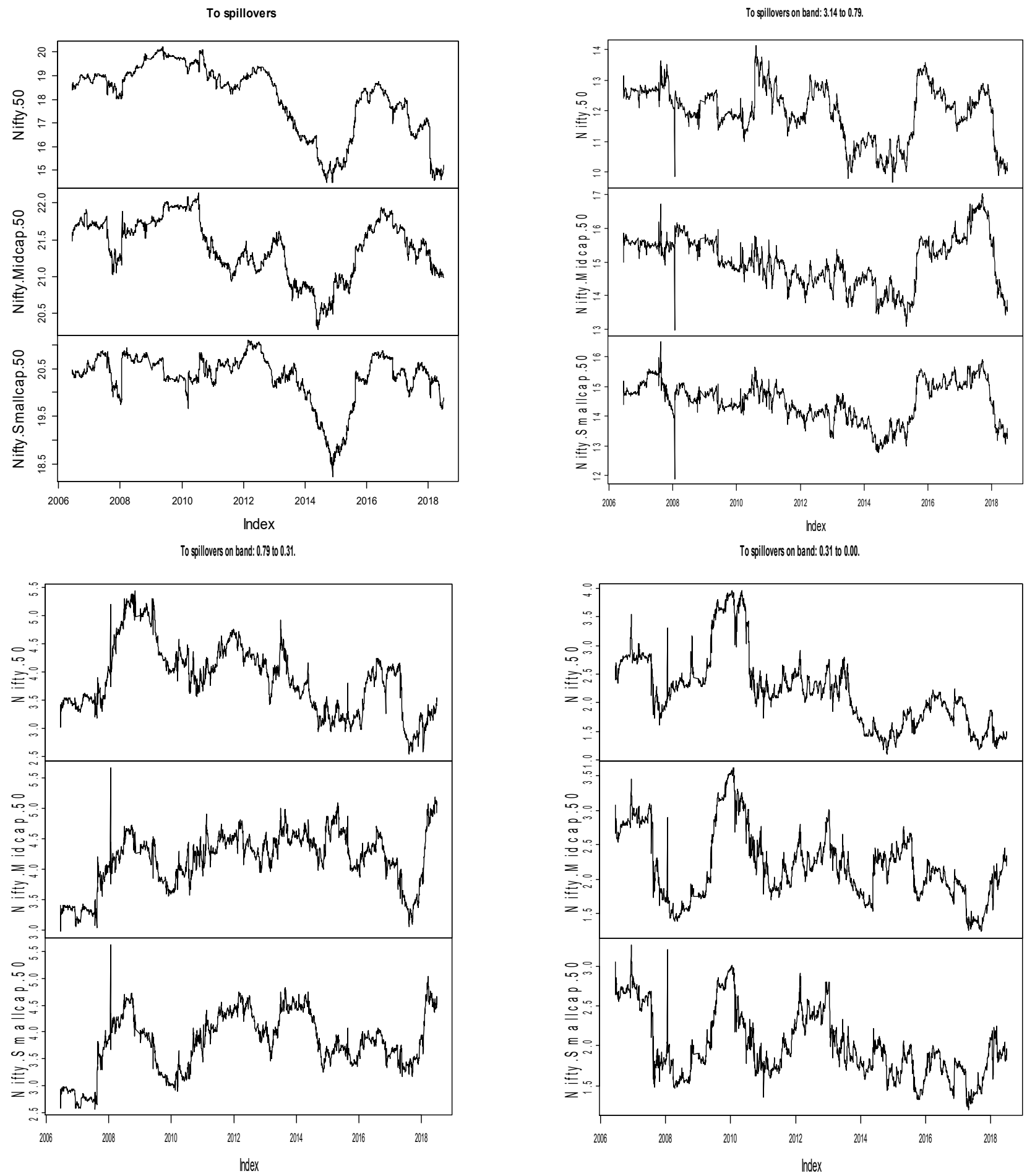

Figure A2. Diebold and Yilmaz (2012) and Baruník and Krehlik (2018) rolling gross volatility spillover to others in the system.

\section{Notes}

Vide the circular SEBI/HO/IMD/DF3/CIR/P/2017/114, the Securities and Exchange Board of India (SEBI) defines large-, mid-, and small-cap stocks as the 1st-100th, 101st-250th companies, and the 251st company onwards, respectively, in terms of full market capitalization.

https:/ / economictimes.indiatimes.com/markets/stocks/news/a-benign-vix-not-reflecting-underlying-fear-on-d-street/articl eshow /64925399.cms?from=mdr (accessed on 12 December 2018). 
3 In terms of dollar-adjusted performance, India has been the most consistent performer, and an important destination for investment for foreign investors. It has been ranked third among the top twenty most followed global indices in terms of 1-year, 2-year, 3-year, and 4-year performances, i.e., a return of $6 \%, 20 \%, 42 \%$, and 21\%, respectively. In terms of the 5 -year and 10 -year performances, it has been ranked second and fifth, with a return of 53\% and 150\%, respectively (source: Bloomberg) ET: 17 April 2019.

4 The BDS test of nonlinearity (Broock et al. 1996) is applied on the residual of a regression estimate in a bilateral context. The results are available upon request.

5 The total pairwise connectedness is obtained for the example in the case of the mid- and small-caps by summing up the volatility crossover in both directions, i.e., 34.06 plus 33.

6 The union government was formed by the Bhartiya Janata Party (BJP) with an absolute majority in the Parliament.

\section{References}

Asli, Ascioglu. 2014. US small-cap indexes: An empirical analysis of factor exposure and return. The Journal of Index Investing 5: 21-32. [CrossRef]

Bai, Ye, and Christopher J. Green. 2010. International diversification strategies: Revisited from the risk perspective. Journal of Banking and Finance 34: 236-45. [CrossRef]

Bai, Jushan, and Pierre Perron. 2003. Computation and analysis of multiple structural change models. Journal of Applied Econometrics 18: 1-22. [CrossRef]

Baruník, Jozef, and Tomáš Krehlik. 2018. Measuring the frequency dynamics of financial connectedness and systemic risk. Journal of Financial Econometrics 16: 271-96. [CrossRef]

Baruník, Jozef, Evžen Kočenda, and Lukáš Vácha. 2016a. Asymmetric connectedness on the US stock market: Bad and good volatility spillovers. Journal of Financial Markets 27: 55-78. [CrossRef]

Baruník, Jozef, Evžen Kočenda, and Lukáš Vácha. 2016b. Gold, oil, and stocks: Dynamic correlations. International Review of Economics and Finance 42: 186-201. [CrossRef]

Baur, Dirk G., and Brian M. Lucey. 2009. Flights and contagion-An empirical analysis of stock-bond correlations. Journal of Financial Stability 5: 339-52. [CrossRef]

Beirne, John, Maria Caporale Guglielmo, Schulze-Ghattas Marianne, and Spagnolo Nicola. 2013. Volatility spillovers and contagion from mature to emerging stock markets. Review of International Economics 21: 1060-75. [CrossRef]

Broock, William A., José Alexandre Scheinkman, W. Davis Dechert, and Blake LeBaron. 1996. A test for independence based on the correlation dimension. Econometric Reviews 15: 197-235. [CrossRef]

Carrieri, Francesca, Vihang Errunza, and Sergei Sarkissian. 2004. Industry risk and market integration. Management Science 50: 207-21. [CrossRef]

Copeland, Maggie, and Thomas Copeland. 2016. VIX versus Size. The Journal of Portfolio Management 42: 76-83. [CrossRef]

Diebold, Francis X., and Kamil Yilmaz. 2009. Measuring financial asset return and volatility spillovers, with application to global equity markets. The Economic Journal 119: 158-71. [CrossRef]

Diebold, Francis X., and Kamil Yilmaz. 2012. Better to give than to receive: Predictive directional measurement of volatility spillovers. International Journal of Forecasting 28: 57-66. [CrossRef]

Elias, Christopher J. 2016. Asset pricing with expectation shocks. Journal of Economic Dynamics and Control 65: 68-82. [CrossRef]

Eun, Cheol S., Wei Huang, and Sandy Lai. 2008. International diversification with large-and small-cap stocks. Journal of Financial and Quantitative Analysis 43: 489-524. [CrossRef]

Ferrer, Román, Syed Jawad Hussain Shahzad, Raquel López, and Francisco Jareño. 2018. Time and frequency dynamics of connectedness between renewable energy stocks and crude oil prices. Energy Economics 76: 1-20. [CrossRef]

Fleming, Jeff, Chris Kirby, and Barbara Ostdiek. 1998. Information and volatility linkages in the stock, bond, and money markets. Journal of Financial Economics 49: 111-37. [CrossRef]

Gorman, Larry. 2003. Conditional performance, portfolio rebalancing, and momentum of small-cap mutual funds. Review of Financial Economics 12: 287-300. [CrossRef]

Huang, Wei. 2007. Financial integration and the price of world covariance risk: Large-vs small-cap stocks. Journal of International Money and Finance 26: 1311-37. [CrossRef]

Kim, Moon K., and David A. Burnie. 2002. The firm size effect and the economic cycle. Journal of Financial Research 25: 111-24. [CrossRef]

Koop, Gary, M. Hashem Pesaran, and Simon M. Potter. 1996. Impulse response analysis in nonlinear multivariate models. Journal of Econometrics 74: 119-47. [CrossRef]

Kumar, Gaurav, and Arun Kumar Misra. 2018. Commonality in liquidity: Evidence from India's national stock exchange. Journal of Asian Economics 59: 1-15. [CrossRef]

Li, Lingfeng. 2002. Macroeconomic Factors and the Correlation of Stock and Bond Returns. Yale ICF Working Paper. New Haven: Yale International Centre for Finance, Department of Economics, Yale University, pp. 2-46. Available online: https://ssrn.com/abstr act $=363641$ (accessed on 10 December 2018). 
Li, Yanan, and David E. Giles. 2015. Modelling volatility spillover effects between developed stock markets and Asian emerging stock markets. International Journal of Finance and Economics 20: 155-77. [CrossRef]

Lin, Pin-Te. 2013. Examining volatility spillover in Asian REIT markets. Applied Financial Economics 23: 1701-5. [CrossRef]

Maghyereh, Aktham I., Hussein Abdoh, and Basel Awartani. 2019. Connectedness and hedging between gold and Islamic securities: A new evidence from time-frequency domain approaches. Pacific-Basin Finance Journal 54: 13-28. [CrossRef]

Mayer, Jörg. 2009. The Growing Interdependence between Financial and Commodity Markets (No. 195). Geneva: United Nations Conference on Trade and Development.

Mensi, Walid, Mobeen Ur Rehman, and Xuan Vinh Vo. 2020. Spillovers and co-movements between precious metals and energy markets: Implications on portfolio management. Resources Policy 69: 101836. [CrossRef]

Moreira, Alan, and Tyler Muir. 2017. Volatility-Managed Portfolios. The Journal of Finance 72: 1611-44. [CrossRef]

Moreira, Alan, and Tyler Muir. 2019. Should Long-Term Investors Time Volatility? Journal of Financial Economics 131: 507-27. [CrossRef]

Müller, Ulrich A., Michel M. Dacorogna, Rakhal D. Davé, Richard B. Olsen, Olivier V. Pictet, and Jacob E. Von Weizsäcker. 1997. Volatilities of different time resolutions-analyzing the dynamics of market components. Journal of Empirical Finance 4: 213-39.

Oberholzer, Niel, and Sven T. von Boetticher. 2015. Volatility spill-over between the JSE/FTSE indices and the South African Rand. Procedia Economics and Finance 24: 501-10. [CrossRef]

Pesaran, H. Hashem, and Yongcheol Shin. 1998. Generalized impulse response analysis in linear multivariate models. Economics Letters 58: 17-29. [CrossRef]

Reinganum, Marc R. 1999. The significance of market capitalization in portfolio management over time. The Journal of Portfolio Management 25: 39-50. [CrossRef]

Semenov, Andrei. 2015. The small-cap effect in the predictability of individual stock returns. International Review of Economics and Finance 38: 178-97. [CrossRef]

Sensoy, Ahmet. 2017. Firm size, ownership structure, and systematic liquidity risk: The case of an emerging market. Journal of Financial Stability 31: 62-80. [CrossRef]

Siegel, Jeremy J. 2021. Stocks for the Long Run: The Definitive Guide to Financial Market Returns E Long-Term Investment Strategies. New York: McGraw-Hill Education.

Switzer, Lorne N. 2010. The behaviour of small cap vs. large cap stocks in recessions and recoveries: Empirical evidence for the United States and Canada. The North American Journal of Economics and Finance 21: 332-46. [CrossRef]

Switzer, Lorne N., and Mingjun Tang. 2009. The Impact of Corporate Governance on the Performance of US Small-Cap Firms. International Journal of Business 14: 341-55.

Tiwari, Aviral Kumar, Juncal Cunado, Rangan Gupta, and Mark E. Wohar. 2018. Volatility spillovers across global asset classes: Evidence from time and frequency domains. The Quarterly Review of Economics and Finance 70: 194-202. [CrossRef]

Underwood, Shane. 2009. The cross-market information content of stock and bond order flow. Journal of Financial Markets 12: 268-89. [CrossRef]

Wang, Xunxiao, and Yudong Wang. 2019. Volatility spillovers between crude oil and Chinese sectoral equity markets: Evidence from a frequency dynamics perspective. Energy Economics 80: 995-1009. [CrossRef]

Wang, Jun, Robert Brooks, Xing Lu, and Hunter M. Holzhauer. 2014. Growth/Value, Market Cap, and Momentum. The Journal of Investing 23: 33-42. [CrossRef]

Zhang, Dayong, and David C. Broadstock. 2018. Global financial crisis and rising connectedness in the international commodity markets. International Review of Financial Analysis 68: 101239. [CrossRef] 